\title{
Association between cervical dysplasia and human papillomavirus in HIV seropositive women from Johannesburg South Africa
}

\author{
Cynthia Firnhaber $\cdot$ Hoa Van Le $\cdot$ Audrey Pettifor $\cdot$ Doreen Schulze • \\ Pam Michelow • Ian M. Sanne • David A. Lewis • Anna-Lise Williamson • \\ Bruce Allan · Sophia Williams • Allen Rinas • Simon Levin · Jennifer S. Smith
}

Received: 4 December 2008 / Accepted: 10 November 2009/Published online: 1 December 2009

(C) The Author(s) 2009. This article is published with open access at Springerlink.com

\begin{abstract}
Objective To examine the association between CD4 counts, HPV infection and the risk of cervical neoplasia among HIV-seropositive women.

Methods A cross-sectional observational study was conducted among 1,010 HIV-seropositive women using cytology-based Pap smears. HPV DNA testing using Linear Array genotyping assay (Roche) was carried out in a subset of 191 patients. Multivariable-adjusted prevalence ratios (mPR) and 95\% confidence intervals (CIs) were estimated with log-binomial regression.

Results Among 1,010 HIV-seropositive women, the prevalence of AGC/ASCUS, LSIL and HSIL or greater was $8.3,23.5$ and $18.0 \%$, respectively. The risk of cervical lesions was higher with $\mathrm{CD} 4<200$ cells $/ \mathrm{mm}^{3}$ vs. CD4 levels $>500 / \mathrm{mm}^{3}$. HPV types $16(41.7 \%)$ and HPV 56
\end{abstract}

C. Firnhaber $(\bowtie)$ D. Schulze - I. M. Sanne

Department of Medicine, Clinical HIV Research Unit,

University of Witwatersrand, Johannesburg, South Africa

e-mail: cfirnhaber@witshealth.co.za

H. Van Le - A. Pettifor · A. Rinas · J. S. Smith $(\bowtie)$

Department of Epidemiology, University of North Carolina

Gillings School of Global Public Health, Campus Box 7435,

Chapel Hill, NC 27599, USA

e-mail: JenniferS@unc.edu

P. Michelow

Cytology Unit National Health Laboratory Service,

Johannesburg, South Africa

D. A. Lewis

STI Reference Centre, National Institute for Communicable Diseases (NHLS), Johannesburg, South Africa

D. A. Lewis

Department of Internal Medicine, University of the

Witwatersrand, Johannesburg, South Africa
(22.2\%) were the most common types in HSIL cases. Women with CD4 levels $<200 / \mathrm{mm}^{3}$ had a higher prevalence of HPV types $16(p<0.01)$ and $66(p=0.04)$. No statistical relationship between cervical lesions and HAART use was found.

Conclusion The burden of HPV infection and HSIL was high and correlated with HIV-induced immunosuppression. HPV 16 was the most common type in HSIL and increased in prevalence with greater immune suppression. Prophylactic HPV 16 vaccination could prevent approximately $40 \%$ of HSIL cases. Strengthening screening programs is imperative in this population.

Keywords Cervical dysplasia - HIV - South Africa . Human papillomavirus

\section{A.-L. Williamson · B. Allan}

Institute of Infectious Disease and Molecular Medicine,

University of Cape Town, Cape Town, South Africa

A.-L. Williamson

National Health Laboratory Service, Groote Schuur Hospital,

Cape Town, South Africa

S. Williams

Right to Care/Pepfar, Johannesburg, South Africa

S. Levin

Department of OB/Gyn, Cornation Hospital,

University of Witswaterand, Johannesburg,

South Africa

P. Michelow

Department of Pathology, University of Witwatersrand,

Johannesburg, South Africa 


\section{Introduction}

Cervical cancer is the second most common female cancer worldwide, and the most common cancer among women in many less developed countries without adequate access to quality screening programs [1]. Oncogenic human papillomavirus (HPV) is a necessary cause of invasive cervical cancer (ICC) [2, 3], with HPV types 16 and 18 being attributable causes in $\sim 70 \%$ of cases [4]. Although most HPV infections are cleared within 6 months to 2 years [5], persistence of HPV infection is consistently associated with an increased risk of high-grade cervical neoplasia and ICC [6].

Globally in 2007, an estimated 33.2 million people were HIV infected; 22.5 million of these people live in subSaharan Africa with 61\% (13.75 million) of these being women [7]. HIV-seropositive women have a notably higher risk of cervical neoplasia and ICC than HIV-seronegative women [8]. HIV-induced immunosuppression has been associated with a higher risk of cervical neoplasia and a higher prevalence of overall and oncogenic type HPV infections [9]. Among HIV-seropositive women, HPV infections are not only more common $[10,11]$, but are more likely to persist $[12,13]$ and consequently result in a higher prevalence of high-grade cervical lesions [14, 15] than among HIV-seronegative women.

Data concerning the relative importance of the most common HPV types in ICC among HIV-seronegative women (type 16) have been inconsistent among HIVseropositive women [4, 16] and [17]. These data have important implications for currently available HPV prophylactic vaccines targeting oncogenic HPV types 16 and 18 [18, 19]. A global review of HPV types in HIVseropositive women found that HPV type 16 prevalence increased with increasing severity of cervical lesions [17], but was less commonly found in high-grade squamous intra-epithelial lesions (HSIL) cases in HIV-seropositive women when compared to HSIL among HIV-seronegative women [4, 17]. In contrast, a case-series from Kenya found a similar proportion of HPV 16 positive among HIVseropositive $(41 \%)$ than HIV-seronegative women $(44 \%)$ with ICC [8]. Among HIV-seropositive women from North America and Europe, HPV 16 prevalence was more weakly associated with immune suppression (as measured by reduced CD4 counts) than other HPV types [9, 20], suggesting that HPV 16 may be better able to evade immune responses than other HPV types. Data are currently lacking, however, on associations between HIV-induced immune suppression and type-specific HPV 16 infection among HIV-seropositive women within the African context.

With the global increase in funding to facilitate the treatment of more HIV-infected individuals in less developed countries, more HIV-infected women are now accessing antiretroviral therapy. Some previous studies have shown a regression of cervical lesions among HIVinfected women receiving highly active antiretroviral therapy (HAART) [21, 22]; however, others have shown no difference between women untreated or treated with different antiretroviral regimens $[14,23]$. One study found that HAART therapy did not appear to significantly affect oncogenic HPV persistence [24], and cervical HPV infection has been shown to persist in a high proportion of patients using HAART [21, 23]. Although HAART for HIV-infected women has a clear effect on restoration of the immune status, the effect of antiretroviral treatment on cervical neoplasia is little known and still debated.

We present here an observational study examining HIVinduced immune suppression (measured by reduced CD4 counts) and other factors on the risk of HPV infection and cervical neoplasia among HIV-seropositive women in South Africa.

\section{Materials and methods}

Study population and enrollment

A baseline, cross-sectional study of HIV-infected women was conducted within the South Africa Cervical Cancer Cohort (SACCC) [25]. HIV-infected women aged from 18 to 65 were recruited from an adult HIV outpatient clinic in a teaching hospital affiliated with the University of Witwatersrand in Johannesburg. Women were eligible to participate in this study unless they (1) were pregnant; (2) had undergone a hysterectomy or conization; (3) were severely ill; or (4) had symptoms and/or signs suggestive of a sexually transmitted infection (STI). Women were study eligible following the treatment of any symptomatic STI, and, if pregnant, 6 weeks after delivery. Of 1,574 women who met eligibility criteria, 66\% agreed to join the study, resulting in 1,039 participating women. After the exclusion of 29 women with cytological data not available, a total of 1,010 HIV-infected women were included.

After an educational session was presented on cervical cancer screening in English or Zulu, women were invited for a Pap smear and to participate in this observational study. Health workers screened for exclusion criteria, explained study aims and obtained written informed consent. A medical history was obtained by participant interview including information on sociodemographics, antiretroviral therapy status and lifestyle factors, including smoking/snuff (traditional chewing tobacco), reproductive/menstrual characteristics, previous Pap smear results if applicable, sexual history/behaviors, history of STIs and contraceptive use. All protocols were reviewed and cleared by the Ethical Committee of the University of Witwatersrand Human Research 
Ethics Committee (Medical) and, for secondary data analysis, from the University of North Carolina.

Gynecological examination, specimen collection and processing

During a pelvic examination, cervical exfoliated cells were collected using two individual endocervical brushes for a conventional Pap smear diagnosis and HPV DNA detection. Conventional cervical smears were performed as liquid-based cytology is currently not available in South Africa. Cytology slides were read and analyzed according to the Bethesda 2001 reporting guidelines [26]. Women with atypical squamous cell-high (ASC-H) and HSIL were referred for immediate colposcopy. Women with ASCUS or low-grade intra-epithelial lesions (LSIL) were followed with a repeat Pap smear after 1 year if their CD4 count was $\geq 200$, or after 6 months if their CD4 count was $<200$ / $\mathrm{mm}^{3}$. Women were also referred for colposcopy if there were 3 consecutive LSIL results over 18 months or greater. For quality control, $10 \%$ of the conventional cytology slides were sent to University of North Carolina for blinded double-reading on two occasions, and a high rate of concordance was observed (81-85\%). HPV brushes were placed in PBS solution and stored in a $4^{\circ} \mathrm{C}$ refrigerator and shipped to University of Cape Town on ice for HPV DNA laboratory testing within 2 weeks of collection.

HIV-seropositive women were treated according to the HIV South African Guidelines on Comprehensive HIV and AIDS Care, Management and Treatment [27], which initiates highly active antiretroviral therapy (HAART) at WHO stage 4 or CD 4 count $\leq 200$ cells $/ \mathrm{mm}^{3}$. The women who had CD4 counts over 500 had originally started HAART according to the above criteria and had good clinical response to treatment.

\section{HPV DNA PCR laboratory testing}

HPV DNA testing was conducted on samples from randomly selected women using the Roche Linear Array HPV genotyping test (Roche Molecular Systems Inc. California USA), and the results were validated utilizing the betaglobin according to the manufacturer's instructions. Laboratory personnel were blinded of all other laboratory and medical history data. HPV DNA results were limited to 191 women ( $\sim 20 \%$ of the study population) due to funding constraints.

The Pap smear was done at the same visit that the HPV DNA was collected from women without any knowledge of any previous Pap smear results, and the woman chosen were the first 191 women who agreed to have the HPV testing done. The Linear Array assay detects a total of 37 HPV types. Individual HPV types were divided into 14 oncogenic (high risk) types: 16, 18, 31, 33, 35, 39, 45,51, 52, 56, 58, 59, 66, 68, and 23 non-oncogenic types (low risk): HPV 6, 11, 26, 40, 42, 53, 54, 55, 61, 62, 64, 67, 69,70, 71, 72, 73, 81, 82, 83, 84, IS39 and CP6108 [28].

Statistical analysis

Current antiretroviral therapy status, sociodemographic, reproductive and sexual behavior characteristics were calculated, stratified by three CD4 count categories $(<200$, $200-500$ and $>500 / \mathrm{mm}^{3}$ ). Age-adjusted prevalence ratios (PRs) for cervical lesions and corresponding 95\% confidence intervals (CIs) were calculated by means of logbinomial regression [29] for each grade of cervical abnormality according to the Bethesda classification system [26] (ASCUS, LSIL, and HSIL or greater). Multivariable-adjusted prevalence ratios (mPR) and 95\% CIs were estimated with log-binomial regression to determine factors associated with cervical lesion outcomes. An initial multivariable model included all potential confounders, and final multivariable model was built using a backward selection method with $10 \%$ change-in-estimate criteria. We systematically choose to retain variables in the final multivariable model if they were significant for any clinical outcome (AGC/ASCUS, LSIL, $\geq$ HSIL) in order to allow the direct comparison of risk factors between different stages of cervical neoplasia. Multivariable models included age categorized into three levels: $<30,30-40$ and $>40$ years. Polychotomous variables were included as sets of indicator variables.

The number and relative percentage of HPV types were calculated among a subgroup of 191 women with available data on HPV DNA, stratified by cervical status and CD4 count grouping. The prevalence of overall HPV, multiple and single oncogenic HPV types was also calculated. Women in the oncogenic group have at least one or more oncogenic types. Relative differences in HPV prevalence were tested using chi-squared statistics using two-sided $p$ values and were not adjusted for other risk factors.

\section{Results}

A total of 1,010 HIV-seropositive women participated, with a median age 34 years (range 18-65). Approximately one-third of women $(32.4 \%)$ reported having graduated from high school. Few women reported ever smoking $(5.5 \%)$ and $20.8 \%$ reported current alcohol use. A high proportion of women $(42.8 \%)$ reported having had five or more lifetime sexual partners. The most commonly reported method of contraception was male condoms $(75.4 \%)$, while $12.4 \%$ reported a history of oral contraceptive use. 
Over two-thirds $(72 \%)$ of women reported having never had a Pap smear. Less than $1 \%$ of women reported a previous history of treatment of cervical dysplasia.

When examining differences by stratified CD4 counts (Table 1), more women with lower CD4 counts $<200$ / $\mathrm{mm}^{3}$ were currently on antiretroviral (HAART) therapy $(74.5 \%)$ than with higher CD4 counts $>500 / \mathrm{mm}^{3}(51.7 \%)$ $(p<0.01)$. Women with CD4 counts $<200 / \mathrm{mm}^{3}$ were also less likely to report current use of oral contraceptives compared to women with CD4 counts $>500 / \mathrm{mm}^{3}$ (9.6 vs. $11.9 \%, p<0.05$ ) (Table 1). Other factors did not appear to be associated with HIV-induced immune suppression, including educational attainment, smoking, alcohol status, age at first intercourse, history of condom use or reported number of sexual partners.
Cervical lesions and other characteristics

The overall prevalence of ASCUS/AGC (atypical glandular cells), LSIL, HSIL or greater was 8.3, 23.5 and $18.0 \%$, respectively. A total of 2 cases of ICC were found, resulting in a prevalence rate of 198/100,000. Lower CD4 count levels after adjusting for age were associated with an increased prevalence of abnormal cervical cytology. Only in the ASCUS/AGC category this trend was not found to be statistically significant: ASCUS/AGC (including 2 cases of AGC $):\left(P R=1.9 ; 95 \%\right.$ CI: $0.9-3.7$ for CD4 $<200 / \mathrm{mm}^{3}$ vs. $\left.>500 / \mathrm{mm}^{3}\right)$, LSIL $(\mathrm{PR}=2.5 ; 95 \% \mathrm{CI}$ : $1.6-4.0$ for CD4 $<200 / \mathrm{mm}^{3}$ vs. $>500 / \mathrm{mm}^{3}$ and HSIL $(\mathrm{PR}=2.5 ; 95 \%$ CI: $1.5-4.4$ for CD4 $<200 / \mathrm{mm}^{3}$ vs. $>500 / \mathrm{mm}^{3}$ )(Table 2). When compared to women under 30 years of age, LSIL was

Table 1 Sociodemographic, sexual behavior and reproductive characteristics of 1,010 HIV-infected women in Johannesburg, South Africa

\begin{tabular}{|c|c|c|c|c|}
\hline & $\mathrm{CD} 4<200 / \mathrm{mm}^{3}$ & CD4 200-500/mm ${ }^{3}$ & $\mathrm{CD} 4>500 / \mathrm{mm}^{3}$ & Overall \\
\hline$n$ & 428 & 464 & 118 & 1,010 \\
\hline Age (median in years, range) & $34(20-57)$ & $34(18-65)$ & $34(19-56)$ & $34(18-65)$ \\
\hline $\mathrm{CD} 4$ count $/ \mathrm{mm}^{3}$ (median, range) & $102(1-199)$ & $304(200-499)$ & $626(501-1,789)$ & $231(1-1,789)$ \\
\hline \multicolumn{5}{|l|}{$\operatorname{HAART}^{\mathrm{a}}(\%)(p$ for difference $<0.001)$} \\
\hline None & 25.5 & 40.5 & 48.3 & 35.1 \\
\hline Regime $1 \mathrm{a}^{\mathrm{b}}$ & 61.7 & 37.7 & 22.9 & 46.1 \\
\hline Regime $1 b^{b}$ & 8.4 & 8.4 & 10.2 & 8.6 \\
\hline Regime $2^{\mathrm{b}}$ & 1.4 & 3.5 & 5.1 & 2.8 \\
\hline Others & 3.0 & 9.9 & 13.6 & 7.4 \\
\hline \multicolumn{5}{|l|}{ Cervical status $(\%)(p$ for difference $<0.001)$} \\
\hline Normal & 39.5 & 55.6 & 67.8 & 50.2 \\
\hline AGC $/$ ASCUS & 9.1 & 7.8 & 7.6 & 8.3 \\
\hline LSIL & 31.8 & 18.1 & 14.4 & 23.5 \\
\hline$\geq$ HSIL $^{\mathrm{d}}$ & 19.6 & 18.5 & 10.7 & 18.0 \\
\hline \multicolumn{5}{|l|}{ Education $(\%)$} \\
\hline Up to grade 8 & 30.4 & 24.1 & 24.6 & 26.8 \\
\hline Grade $8-12$ & 39.3 & 43.5 & 35.6 & 40.8 \\
\hline$\geq 12$ & 30.4 & 32.3 & 39.8 & 32.4 \\
\hline Ever smoking $(\%)$ & 4.2 & 7.1 & 4.2 & 5.5 \\
\hline Current alcohol use (\%) & 18.0 & 23.3 & 21.2 & 20.8 \\
\hline \multicolumn{5}{|l|}{ Age at first intercourse (\%) } \\
\hline$<15$ years & 11.5 & 11.2 & 9.3 & 11.1 \\
\hline $15-18$ years & 58.4 & 55.8 & 55.9 & 56.9 \\
\hline$\geq 19$ years & 30.1 & 33.0 & 34.8 & 32.0 \\
\hline Number of lifetime sexual partners $\geq 5\left(\%^{\mathrm{e}}\right)$ & 45.0 & 42.6 & 35.6 & 42.8 \\
\hline Parity (median, range) & $2(0-9)$ & $2(0-7)$ & $2(0-7)$ & $2(0-15)$ \\
\hline Current oral contraceptive use $(\%)(p$ for difference $<0.05)$ & 9.6 & 15.1 & 11.9 & 12.4 \\
\hline Current condom use $(\%)$ & 80.0 & 76.9 & 81.4 & 75.4 \\
\hline
\end{tabular}

${ }^{a}$ HAART-highly active antiretroviral therapy

b 1a Stavudine, Lamivudine, Efavirenz; 1b Stavudine, Lamivudine, Nevirapine; 2 Zidovudine, Didanosine, Lopinavir/Ritonavir

c AGC/ASCUS include 2 cases of AGC

${ }^{\mathrm{d}} \geq$ HSIL includes 2 cases of ICC

${ }^{\text {e }}$ Denominators for percentages excluded observations with missing values 
Table 2 Risk factors and prevalence ratios among 1,010 HIV-infected women in Johannesburg, South Africa

\begin{tabular}{|c|c|c|c|c|c|c|c|}
\hline & \multirow{2}{*}{$\begin{array}{l}\text { Normal } \\
n=507\end{array}$} & \multicolumn{2}{|c|}{$\mathrm{AGC} / \mathrm{ASCUS}^{\mathrm{a}}$} & \multicolumn{2}{|r|}{ LSIL } & \multicolumn{2}{|c|}{$\geq \mathrm{HSIL}^{\mathrm{b}}$} \\
\hline & & $n=84$ & $\mathrm{PR}^{\mathrm{c}}(95 \% \mathrm{CI})$ & $n=237$ & $\mathrm{PR}^{\mathrm{c}}(95 \% \mathrm{CI})$ & $n=182$ & $\mathrm{PR}^{\mathrm{c}}(95 \% \mathrm{CI})$ \\
\hline \multicolumn{8}{|c|}{ Age (years) } \\
\hline$<30^{\mathrm{d}}$ & 106 & 27 & 1 & 74 & 1 & 38 & 1 \\
\hline $30-40$ & 267 & 40 & $0.6(0.4-1.0)$ & 120 & $0.8(0.6-0.9)$ & 103 & $1.1(0.8-1.5)$ \\
\hline$>40$ & 134 & 17 & $0.6(0.3-1.0)$ & 43 & $0.6(0.4-0.8)$ & 41 & $0.9(0.6-1.3)$ \\
\hline \multicolumn{8}{|c|}{$\mathrm{CD} 4$ count $/ \mathrm{mm}^{3}$} \\
\hline$<200$ & 169 & 39 & $1.9(0.9-3.7)$ & 136 & $2.5(1.6-4.0)$ & 84 & $2.5(1.5-4.4)$ \\
\hline $200-500$ & 258 & 36 & $1.2(0.6-2.5)$ & 84 & $1.4(0.9-2.3)$ & 86 & $1.9(1.1-3.3)$ \\
\hline \multirow[t]{2}{*}{$>500^{\mathrm{d}}$} & 80 & 9 & 1 & 17 & 1 & 12 & 1 \\
\hline & & & $p$ for trend $<0.01$ & & $p$ for trend $<0.001$ & & $p$ for trend $<0.001$ \\
\hline \multicolumn{8}{|c|}{ Education attainment (grade) } \\
\hline$<8^{\mathrm{d}}$ & 147 & 22 & 1 & 54 & 1 & 48 & 1 \\
\hline $8-12$ & 198 & 31 & $1.0(0.9-1.1)$ & 102 & $0.9(0.8-1.1)$ & 81 & $0.9(0.9-1.1)$ \\
\hline$>12$ & 162 & 31 & $1.0(0.9-1.1)$ & 81 & $1.0(0.8-1.1)$ & 53 & $1.0(0.9-1.1)$ \\
\hline \multicolumn{8}{|c|}{ Current alcohol use } \\
\hline $\mathrm{No}^{\mathrm{d}}$ & 393 & 61 & 1 & 187 & 1 & 159 & 1 \\
\hline Yes & 114 & 23 & $1.0(0.9-1.1)$ & 50 & $1.0(0.9-1.1)$ & 23 & $1.2(1.1-1.3)$ \\
\hline \multicolumn{8}{|c|}{ Age at first intercourse (years) } \\
\hline$\geq 19^{\mathrm{d}}$ & 180 & 21 & 1 & 71 & 1 & 51 & 1 \\
\hline $15-18$ & 267 & 52 & $1.6(1.0-2.6)$ & 146 & $1.2(1.0-1.6)$ & 110 & $1.3(1.0-1.8)$ \\
\hline$<15$ & 60 & 11 & $1.4(0.7-2.8)$ & 20 & $0.8(0.5-1.3)$ & 21 & $1.1(0.7-1.8)$ \\
\hline \multicolumn{8}{|c|}{ Number of lifetime sexual partners ${ }^{\mathrm{e}}$} \\
\hline$<5^{\mathrm{d}}$ & 281 & 40 & 1 & 140 & 1 & 114 & 1 \\
\hline$\geq 5$ & 221 & 44 & $1.4(0.9-2.0)$ & 97 & $0.9(0.8-1.2)$ & 68 & $0.8(0.6-1.1)$ \\
\hline \multicolumn{8}{|l|}{ Parity } \\
\hline 0 & 78 & 13 & $1.0(0.6-1.9)$ & 30 & $1.0(0.6-1.1)$ & 13 & $0.6(0.3-1.0)$ \\
\hline $1^{\mathrm{d}}$ & 164 & 24 & 1 & 84 & 1 & 51 & 1 \\
\hline$\geq 2$ & 265 & 47 & $1.4(0.8-2.2)$ & 123 & $1.0(0.8-1.3)$ & 118 & $1.4(1.0-1.8)$ \\
\hline \multicolumn{8}{|c|}{ Current oral contraceptive use } \\
\hline $\mathrm{No}^{\mathrm{d}}$ & 442 & 72 & 1 & 216 & 1 & 155 & 1 \\
\hline Yes & 65 & 12 & $1.0(0.9-1.1)$ & 21 & $1.1(1.0-1.3)$ & 27 & $1.0(0.8-1.1)$ \\
\hline \multicolumn{8}{|c|}{ Current condom use } \\
\hline $\mathrm{No}^{\mathrm{d}}$ & 117 & 20 & 1 & 54 & 1 & 58 & 1 \\
\hline Yes & 390 & 64 & $0.9(0.5-1.4)$ & 183 & $0.9(0.7-1.2)$ & 124 & $0.7(0.5-0.9)$ \\
\hline
\end{tabular}

a AGC/ASCUS include 2 cases of AGC

$\mathrm{b} \geq$ HSIL includes 2 cases of ICC

c Age-adjusted prevalence ratio (PR) vs. 507 HIV-seropositive women with normal cervical status

${ }^{\mathrm{d}}$ Reference category

e Denominators for percentages exclude observations with missing values

also less prevalent among women 30-40 years of age $(\mathrm{PR}=0.8 ; 95 \%$ CI: $0.6-0.9)$ and women over 40 (PR $=0.6 ; 95 \%$ CI: 0.4-0.8). Both ASCUS and HSIL were also less likely among women over age 40, although associations were not statistically significant (Table 2). Approximately, one-third of women with LSIL (31.2\%, $74 / 237)$ and one-fifth of women with HSIL $(20.9 \%, 38 / 182)$ were 30 years or younger.
Current alcohol use was associated with slightly higher prevalence of HSIL (age-adjusted PR $=1.2$; 95\% CI: 1.1 1.3) (Table 2). Age-adjusted prevalence of abnormal cervical cytology was not significantly associated with the sexual history such as age at first intercourse, number of life time sexual partners and higher parity. However, abnormal cytology prevalence appeared to be lower for women who reported current condom use vs. non-users for 
HSIL cases $(\mathrm{PR}=0.7 ; 95 \%$ CI: $0.5-0.9$ for HSIL (Table 2). A history of smoking, using snuff or current oral contraceptive use did not show any association with abnormal cervical cytology (Table 1 ).

In the multiple log-binomial regression models, lower CD4 count ( $<200$ vs. $>500 / \mathrm{mm}^{3}$ ) was consistently associated with all grades of cervical neoplasia: HSIL $(\mathrm{mPR}=2.4 ; 95 \% \mathrm{CI}: 1.4-4.2)$, LSIL $(\mathrm{mPR}=2.4 ; 95 \%$ CI: $1.5-3.8)$ and ASCUS (mPR $=1.8 ; 95 \% \mathrm{CI}: 0.9-3.6)$ (Table 3). Associations between current HAART use were not statistically significant in the multivariate model $(\mathrm{mPR}=1.3 ; 95 \% \mathrm{CI}: 0.9-1.7$ for HSIL; $\mathrm{mPR}=1.2 ; 95 \%$ CI: $0.9-1.5$ for LSIL; $\mathrm{PR}=1.3$; 95\% CI: $0.8-1.9$ for ASCUS).

Associations between HPV infection, cervical lesions and CD4 count

Among the 191 women with HPV DNA results, the prevalence and distribution of abnormal lesions were the same as the main cohort: AGC/ASCUS $7.3 \%$ (14/191), LSIL $27 \%$ (52/191) and HSIL 18.3\% (35/191). There was no statistical difference between the subgroup of women with HPV typing and the main cohort in terms of age, CD4 count, HAART use, educational level, age of first sexual encounter, number of lifetime sexual partners, condom use or smoking habits.

There was a higher prevalence of overall $(p<0.01)$, single $(p<0.01)$ and multiple oncogenic HPV types $(p<0.01)$ with increasing grade of cervical abnormalities.
The prevalence of HPV $16(p<0.01), 56(p<0.01)$, HPV $33(p=0.03)$, HPV $59(p=0.06)$ and HPV $66(p=0.01)$ was also higher in HIV-seropositive women with HSIL or LSIL compared to those with HIV-seropositive women with normal or ASCUS diagnoses (Fig. 1). Compared to women with normal cervical cytology, women with LSIL, HSIL or AGUS/ASCUS had a higher prevalence of any HPV positivity (HSIL: 88.9\%, LSIL: 98.0\%, AGUS/ ASCUS: $100 \%$ and normal: $74.4 \%, p<0.01$ ), any oncogenic HPV (HSIL: 77.8\%, LSIL: 90.2\%, AGUS/ASC 71\%, normal: $60.0 \%, p<0.01)$ or multiple oncogenic HPV types (HSIL: $58.3 \%$, LSIL 68.6\%, AGUS/ASC $42.9 \%$ normal: $38.9 \%, p<0.01)$. The prevalence of oncogenic and multiple HPV types appeared to be generally similar in LSIL and HSIL cases. However, the absolute number of HPV types found in LSIL appeared to be higher in that in HSIL cases (Fig. 2).

HPV type 16 appeared to be more prevalent in more advanced cervical dysplasia (HSIL: $41.7 \%$, 95\% CI: 24.8 $58.6 \%$ (15/36 women), LSIL: $37.3 \%$, 95\% CI: 23.5-51.0\%, (19/51 women), ASCUS: $14.3 \%, 95 \%$ CI: $0.0-35.3 \%(2 / 14$ women) and normal: 17.8\%, 95\% CI: 9.7-25.8\% (16/90 women) $(p=0.002)$. In women with negative cytology results, a significant association was seen between CD4 count and HPV 16 with CD4 <200: 27.3\% (9/33), 200500: $15.2 \%(7 / 46)$ and >500: $0 \%(0 / 11), p=0.03$. HPV type 18 was relatively less common in HSIL $(2.8 \%, 95 \%$ CI: $0.0-8.4 \%$ ). No significant difference in HPV positivity by grade of cervical lesion was found for any other individual HPV type. The most common oncogenic HPV types

Table 3 Risk factors and multivariate prevalence ratios among 1,010 HIV-infected women in Johannesburg, South Africa

\begin{tabular}{|c|c|c|c|c|c|c|c|}
\hline & \multirow{2}{*}{$\begin{array}{l}\text { Normal } \\
n=507\end{array}$} & \multicolumn{2}{|c|}{ AGC $/ A S C U S$} & \multicolumn{2}{|c|}{ LSIL } & \multicolumn{2}{|c|}{$\geq$ HSIL $^{\mathrm{b}}$} \\
\hline & & $n=84$ & $\mathrm{mPR}^{\mathrm{c}}(95 \% \mathrm{CI})$ & $n=237$ & $\mathrm{mPR}^{\mathrm{c}}(95 \% \mathrm{CI})$ & $n=182$ & $\mathrm{mPR}^{\mathrm{c}}(95 \% \mathrm{CI})$ \\
\hline \multicolumn{8}{|l|}{ Age } \\
\hline$<30$ years $^{\mathrm{d}}$ & 106 & 27 & 1 & 74 & 1 & 38 & 1 \\
\hline $30-40$ years & 267 & 40 & $0.7(0.4-1.0)$ & 120 & $0.8(0.6-0.9)$ & 103 & $1.0(0.7-1.4)$ \\
\hline$>40$ years & 134 & 17 & $0.5(0.3-1.0)$ & 43 & $0.6(0.4-0.8)$ & 41 & $0.8(0.6-1.2)$ \\
\hline \multicolumn{8}{|c|}{$\mathrm{CD} 4$ count $/ \mathrm{mm}^{3}$} \\
\hline$<200$ & 169 & 39 & $1.8(0.9-3.6)$ & 136 & $2.4(1.5-3.8)$ & 84 & $2.4(1.4-4.2)$ \\
\hline $200-500$ & 258 & 36 & $1.2(0.6-2.4)$ & 84 & $1.4(0.9-2.2)$ & 86 & $1.9(1.1-3.3)$ \\
\hline$>500^{\mathrm{d}}$ & 80 & 9 & 1 & 17 & 1 & 12 & 1 \\
\hline \multicolumn{8}{|l|}{ HAART $^{\mathrm{e}}$} \\
\hline $\mathrm{No}^{\mathrm{d}}$ & 201 & 27 & 1 & 71 & 1 & 55 & 1 \\
\hline Yes & 306 & 57 & $1.3(0.8-1.9)$ & 166 & $1.2(0.9-1.5)$ & 127 & $1.3(0.9-1.7)$ \\
\hline
\end{tabular}

${ }^{a}$ AGC/ASCUS include 2 cases of AGC

$\mathrm{b} \geq$ HSIL includes 2 cases of ICC

c Multivariate prevalence ratio (mPR) adjusted for all variables listed in the table vs. 507 HIV-seropositive women with normal cervical status

${ }^{\mathrm{d}}$ Reference category

e HAART—highly active antiretroviral therapy: 1a Stavudine, Lamivudine, Efavirenz; 1b Stavudine, Lamivudine, Nevirapine; 2 Zidovudine, Didanosine, Lopinavir/Ritonavir 


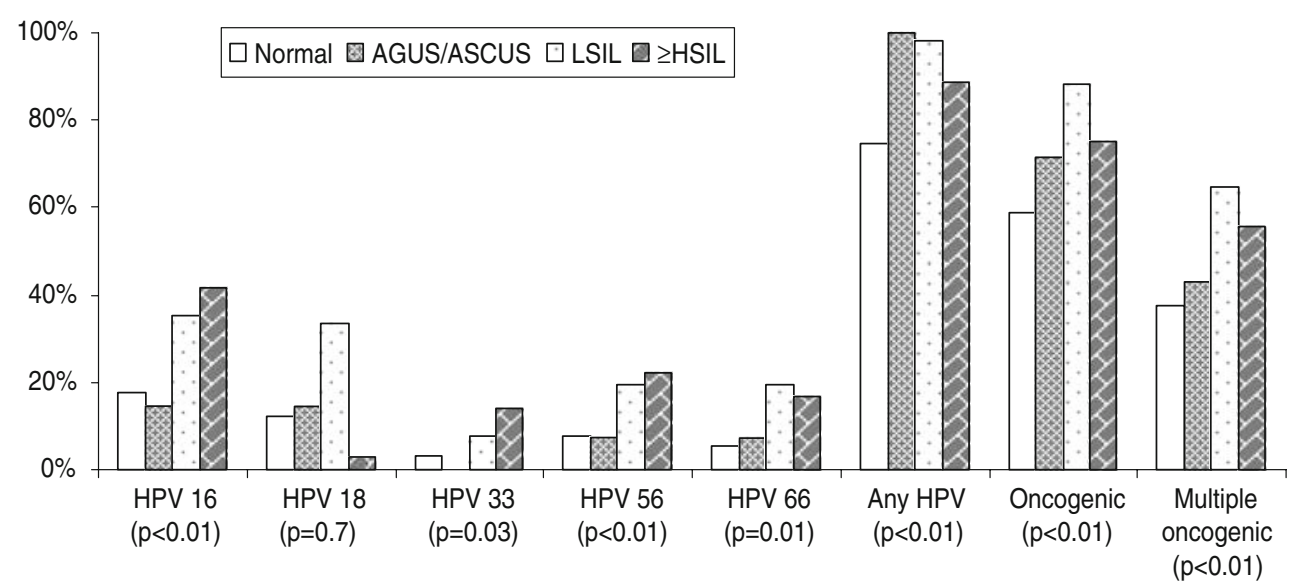

Fig. 1 Prevalence of HPV types by cervical status among 191 HIV-infected women in Johannesburg, South Africa (Normal = 90 women, AGUS/ASCUS $=14$ women, $\mathrm{LSIL}=51$ women and HSIL $=36$ women)

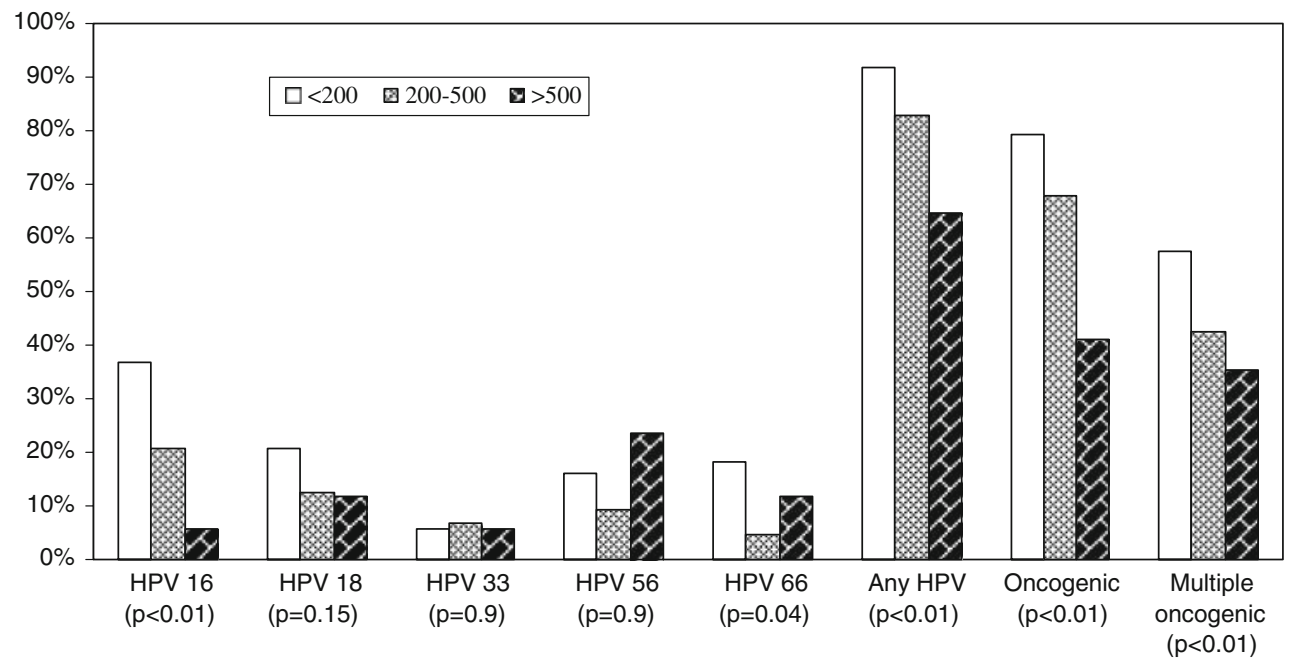

Fig. 2 Prevalence of HPV types by CD4 count levels among 191 HIV-infected women in Johannesburg, South Africa $\left(\right.$ CD4 $<200 / m^{3}$ :

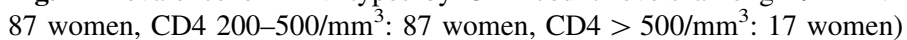

found in women with HSIL lesions or greater were HPV type 16 (41.7\%), HPV 56 (22.2\%), HPV type 66 (16.7\%), HPV type 33 (13.9\%) and HPV type 59 (11.1\%), whereas in LSIL, the most common types included HPV types 16 and 18 (each at $35.3 \%$ ), HPV 56 and 66 (each at 19.6\%) and $61(23.5 \%)$. Combined prevalence of HPV 16 and/or 18 were $41.7 \%$ for HSIL, $52.9 \%$ for LSIL, $28.9 \%$ for women with normal diagnoses $(p=0.02)$.

HIV-seropositive women with CD4 levels $<200 / \mathrm{mm}^{3}$ had higher prevalence of overall HPV types $\{92.0 \%$ (80/ $87)$ vs. $64.7 \%$ (11/17) for $>500$ cells $\left./ \mathrm{mm}^{3}, p<0.01\right\}$, any oncogenic HPV type $\{81.6 \%$ (71/87) vs. $41.2 \%(7 / 17)\}$ for CD4 counts $>500$ cells $/ \mathrm{mm}^{3}, \quad p<0.01$ and multiple oncogenic HPV types $\{59.8 \%$ (52/87) vs. $35.3 \%$ (6/17), $p<0.01\}$. The prevalence of HPV type 16 was also more common among women with lower CD4 counts $\{37.9 \%$ (33/87) for $<200 / \mathrm{mm}^{3}$ vs. $5.9 \%$ (1/17) for $>500 / \mathrm{mm}^{3}$, $p<0.01\}$, as well as HPV type $66\{18.4 \%(16 / 87)$ vs. $11.8 \%(2 / 17), p=0.04\}$.

\section{Discussion}

This cervical cancer study in Johannesburg, South Africa is, to our knowledge, the largest to date among HIVseropositive women in Africa. Half of 1,010 HIVseropositve women had cervical lesions, with cervical abnormalities prevalence increasing with lower CD4 immune status. High observed prevalence of ASCUS or greater is similar to that observed in $397 \mathrm{HIV}$-seropositive women from Cape Town, South Africa (54\%) [30], yet somewhat lower than among $150 \mathrm{HIV}$-seropositive women from Zambia (76\%) [30]. Overall observed prevalence of LSIL (24\%) and HSIL (18\%) was also higher than among 
HIV-seropositive women in the United States (15.4 and $7.9 \%$, respectively), a European cohort (21.0 and $2.8 \%$, respectively) [32, 33] or Zimbabwe (9.7 and 3.4\%) [34].

In this study from Johannesburg, $42 \%$ of HIV-seropositive women had CD4 counts $<200$ cells $/ \mathrm{mm}^{3}$. Our results of HSIL prevalence of $18 \%$ are not as high as that observed in Zambia (33\%) among a smaller number of HIVseropositive women who also had low median CD4 counts of $165 / \mathrm{mm}^{3}$, [31]. Observed prevalence, however, was higher than among women with unknown HIV serostatus screened in the Free State province, South Africa (LSIL of $18 \%$ and HSIL of $8 \%$ ) [35]. The high prevalence of cervical neoplasia in our study could be partially explained by more advanced stages of HIV immunosuppression among female participants when compared to previous studies from the United States and Europe [32, 33]. In addition, the lack of cervical cancer screening is also a likely cause of this phenomenon since cervical cancer in non-HIV-infected women is higher in South Africa than the United States. Of note, one-fifth of HSIL cases were 30 years of age or younger. Given these results, cervical cancer screening should be considered in HIV-seropositive women upon diagnosis rather than being delayed until 30 years of age [36].

The number of different types of any HPV or oncogenic HPV DNA types among HIV-seropositive women was smaller with increasing severity of cervical neoplasia (i.e. HIV-seropositive women with HSIL appear to have fewer HPV types than those with LSIL). These findings are consistent with data among largely HIV-seronegative women [4, 17], indicative of the relatively fewer number of HPV types that may be etiologically important for the development of HSIL vs. lower grades of cervical neoplasia in HIV-seropositive women. Our results, however, are limited to HPV DNA detection within cervical exfoliated cells, rather than biopsy specimens. Although cytological results were presented in current analyses, as used in current clinical practice, histological confirmation of study outcomes may have lead to the reclassification of some clinical endpoints. Of the 182 cases of HSIL or greater, 83 had available pathology results. Most HSIL cases were histologically confirmed as CIN-2 (30\%) or CIN-3 (47\%), whereas slightly less than one quarter $(23 \%)$ were classified as CIN-1 by histology. Measures of association between risk factors and grades of cervical neoplasia presented, respectively, in Tables 2 and 3, were similar, however, when CIN, rather than SIL, classifications were used.

HPV 16 was the most common HPV type in HIVseropositive women with HSIL $(41.7 \%)$ in the present study, with HPV 18 being relatively rare $(2.8 \%)$. These results are similar to a review of African data among largely HIVseronegative women where HPV 16 and/or 18 prevalence was $45 \%$ in HSIL [4], Among 77 HIV-seropositive women with HSIL from Zambia [31], HPV 52 was the most common type, followed by HPV types 58. A previous review indicated that HSIL cases among HIV-seropositive women may have a lower proportion of HPV-16 positivity than HIV-seronegative HSIL cases [17]. Given recent data from Kenya indicating that HPV 16 prevalence was similar in HIV-seropositive and HIV-seronegative ICC cases [8], further data on HPV oncogenic types in HSIL and ICC cases are needed among a larger number of HIV-seropositive HSIL and ICC cases from Africa.

Combined HPV 16 and/or 18 prevalence among HIVseropositive women in South Africa was 42\% HSIL and $53 \%$ in LSIL, respectively. Thus, as with HIV-seronegative women, a notable proportion of HSIL and LSIL cases in HIV-seropositive women could be potentially prevented by the vaccination of female adolescents prior to first sexual intercourse. Not withstanding, a non-negligible proportion of HSIL and LSIL cases will not be prevented by HPV prophylactic vaccination, highlighting that cervical cancer screening remains paramount for optimal cervical cancer prevention. Further, approximately half of HSIL cases among HIV-seropositive women in this study harbored other high-risk HPV types 33, 56 and 66 ( 47\%). This is important to consider for the development of future therapeutic vaccines that are urgently needed in regions with a high burden of ICC.

Among HIV-seropositive women from Johannesburg, lower CD4 counts were consistently associated with a higher risk of cervical lesions (ASCUS, LSIL and HSIL or greater). Our results are consistent with previous screening studies of HIV-seropositive women [37, 38]. It is not surprising that HIV-seropositive women with greater immunosuppression are at a higher risk of cervical disease. Consistently, lower CD4 counts ( $<500$ cells $\left./ \mathrm{mm}^{3}\right)$ have been associated with a higher probability of progression to higher cervical disease grades $[32,37]$.

The relatively broader distribution of HPV types among HIV-seropositive women with lower CD4 counts suggests reactivation of latent HPV viral infections [38]. Our results are similar to previous studies indicating that HIVseropositive women with CD4 counts $<200 / \mathrm{mm}^{3}$ have a higher prevalence of any HPV or oncogenic HPV types [11, 39, 40] when compared to those with CD4 counts $>500 / \mathrm{mm}^{3}$. Of interest, HPV 16 prevalence in our study increased with greater immune suppression, declining from $38 \%$ among women with CD4 counts $<200$ cells/ $\mathrm{mm}^{3}$ to $6 \%$ for $\mathrm{CD} 4$ counts $>500$ cells $/ \mathrm{mm}^{3}$. As a sensitivity analysis, we limited analyses to HIV-seropositive women with normal cytology and found similar results. Albeit based on relatively smaller sample sizes, these results suggest that within the African content that HPV 16 may not be better at evading host immune responses than other HPV types, as previously suggested [6, 9]. Strickler 
et al. showed a relatively weaker association of type 16 with decreasing CD4 counts than other HPV types [9] among HIV-seropositive women from the United States consistently, Koshiol et al. found that the persistence of HPV 16 among HIV-seropositive women did not appear to be associated with CD4 counts [6]. These results from South Africa, although based on relatively small sizes, suggest that HPV 16 prevalence may be affected by the level of CD4 immune suppression. As previously hypothesized, the relationship of HPV 16 infection with the immune suppression in our population may differ from European and US HIV-infected women, potentially due to higher levels of immunosuppression in the underlying population within the African context [9]. Further data are needed to investigate the prevalence and persistence of HPV 16 and other high-risk HPV types, stratified by the level of CD4 count, in HIV-seropositive women in both African and relatively more developed populations.

Another interesting finding in this study was that HIVseropositive women who used condoms had a lower risk of HSIL than non-users (table 2): $\mathrm{PR}=0.795 \% \mathrm{CI}(0.5-0.9)$. A study of HIV-seronegative women $(n=82)$ also found a lower risk of cervical neoplasia among women who reported consistent condom use compared with those who did not [41]. There is also evidence in HIV-seronegative women that the consistent use of condoms was associated with a higher clearance rate of HPV and of cervical neoplasia [42]. In our present study, a protective effect was not found with ASCUS or LSIL with condom use.

Given the cross-sectional design, the current study can not reliably address the temporal effect of HAART on HPV persistence or the progression of cervical neoplasia. The multivariate analysis did not find any association between HAART use and any grade of cervical neoplasia, and is in agreement with previous research [14, 23]. One study among 328 US women found no difference in cervical disease prevalence between HIV-seropositive women treated and untreated with either mono- or combination therapy (non-HAART) over study follow-up [14]. An Italian study of $163 \mathrm{HIV}$-seropositive women also found no beneficial effect of HAART therapy on the risk of incident SIL, or on the progression rate of cervical lesions after adjusting for CD4 cell count [23]. Given inconsistent associations between HAART use and the risk of cervical neoplasia, the effect of HAART therapy on cervical neoplasia is still being debated. Although potent anti-HIV regimens are effective for the restoration of patient's immune system by increasing CD4 counts, limited data suggest that HAART use may not affect HPV viral persistence [21, 23]. Further prospective studies in this cohort will be done to evaluate whether HAART has any role in modifying the progression of cervical dysplasia in these HIV-seropositive women. Further studies are also needed to determine whether the earlier initiation of HAART at higher CD4 counts than is currently recommended for clinical practice will useful for the prevention of highgrade cervical lesions among HIV-seropositive women.

One limitation of this study is the lack of HIV viral load data and analysis as a measure of HIV disease status. HIV baseline viral loads before the initiation of HAART are generally not done in the South African government HIV treatment clinics [27]. Another possible bias in the study is that very ill women were excluded from the study. These women might have had lower $\mathrm{CD}_{4}$ counts; therefore, we may actually be underestimating the prevalence of highgrade lesions that would have been found if these women had not been excluded. However, we do not think that this was a significant selection bias, given that women with $\mathrm{CD}_{4}$ counts $<200$ represents $43 \%$ of our study population.

Given that many African HIV-seropositive women are living longer in the era of HAART, they now face longerterm HIV-related complications including invasive cervical cancer. The wide-spread introduction of currently available prophylactic HPV vaccines would reduce, but not eliminate, a large proportion of high-grade cervical lesions. Thus, strengthening and expanding cervical cancer screening program in settings where HIV prevalence is high remains imperative.

Acknowledgments This study was supported by Cooperative Agreement Number U62/CCU022901 from the Centers for Disease Control and Prevention (CDC), Atlanta, USA and Center for Aids Research University of North Carolina Development Award March 2006. Its contents are solely the responsibility of the authors and do not necessarily represent the official views of the Department of Health and Human Services at the CDC and the National Centre for Global AIDS Prevention (NCHSTP). Preparation of this article was funded in part by a fellowship grant from the Vietnam Education Foundation (VEF) to L. V. Hoa. The opinions, findings and conclusions stated herein are those of the authors and do not necessarily reflect those of VEF.

Open Access This article is distributed under the terms of the Creative Commons Attribution Noncommercial License which permits any noncommercial use, distribution, and reproduction in any medium, provided the original author(s) and source are credited.

\section{References}

1. Parkin DM, Bray F (2006) Chapter 2: the burden of HPV-related cancers. Vaccine 24(Suppl 3):S11-S25

2. Bosch FX, Lorincz A, Munoz N, Meijer CJ, Shah KV (2002) The causal relation between human papillomavirus and cervical cancer. J Clin Pathol 55:244-265

3. Walboomers JM, Jacobs MV, Manos MM et al (1999) Human papillomavirus is a necessary cause of invasive cervical cancer worldwide. J Pathol 189:12-19

4. Smith JS, Lindsay L, Hoots B et al (2007) Human papillomavirus type distribution in invasive cervical cancer and high grade cervical lesions: a meta-analysis update. Int J Cancer 121:621-632 
5. Ho GY, Bierman R, Beardsley L, Chang CJ, Burk RD (1998) Natural history of cervicovaginal papillomavirus infection in young women. N Engl J Med 338(7):423-428

6. Koshiol JE, Lindsay L, Pimenta JM, Poole CL, Jenkins D, Smith JS (2008) Persistent human papillomavirus infection and cervical neoplasia: a systematic review and meta-analysis. Am J Epidemiol 168(2):123-137

7. http://data.unaids.org/pub/EPISlides/2007/071118_epi_regional $\% 20$ factsheet_en.pdf website accessed 20 August 2009

8. De Vuyst H, Gichangi P, Estambale B, Njuguna E, Franceschi S, Temmerman M (2008) Human papillomavirus types in women with invasive cervical carcinoma by HIV status in Kenya. Int $\mathbf{J}$ Cancer 122:244-246

9. Strickler HD, Palefsky JM, Shah KV et al (2003) Human papillomavirus type 16 and immune status in human immunodeficiency virus-seropositive women. J Natl Cancer Inst 95: 1062-1071

10. De Vuyst H, Steyaert S, van Renterghem L et al (2003) Distribution of human papillomavirus in a family planning population in Nairobi, Kenya. Sex Transm Dis 30(2):137-142

11. Palefsky JM, Minkoff H, Kalish LA et al (1999) Cervicovaginal human papillomavirus infection in human immunodeficiency virus-1 (HIV)-positive and high-risk HIV-negative women. J Natl Cancer Inst 91:226-236

12. Minkoff H, Feldman J, DeHovitz J, Landesman S, Burk R (1998) A longitudinal study of human papillomavirus carriage in human immunodeficiency virus-infected and human immunodeficiency virus-uninfected women. Am J Obstet Gynecol 178:982-986

13. Moscicki AB, Ellenberg JH, Farhat S, Xu J (2004) Persistence of human papillomavirus infection in HIV-infected and -uninfected adolescent girls: risk factors and differences, by phylogenetic type. J Infect Dis 190:37-45

14. Ellerbrock TV, Chiasson MA, Bush TJ et al (2000) Incidence of cervical squamous intraepithelial lesions in HIV-infected women. JAMA 283:1031-1037

15. Hawes SE, Critchlow CW, Faye Niang MA et al (2003) Increased risk of high-grade cervical squamous intraepithelial lesions and invasive cervical cancer among African women with human immunodeficiency virus type 1 and 2 infections. J Infect Dis 188:555-563

16. Strickler HD, Palefsky JM, Burk RD (2008) HPV types present in invasive cervical cancers of HIV-seropositive women. Int $\mathrm{J}$ Cancer 123:1224-1225

17. Clifford GM, Goncalves MA, Franceschi S (2006) HPV and HIV study group. Human papillomavirus types among women infected with HIV: a meta-analysis. AIDS 20:2337-2344

18. Harper D, Franco EL, Wheeler C et al (2004) Efficacy of a bivalent $\mathrm{L} 1$ virus-like particle vaccine in prevention of infection with human papillomavirus types 16 and 18 in young women: a randomised controlled trial. Lancet 364(9447):1757-1765

19. Villa LL, Costa RL, Petta CA et al (2005) Prophylactic quadravalent human papillomavirus (types $6,11,16$, and 18) L1 viruslike particle vaccine in young women: a randomised double-blind placebo-controlled multicentre phase II efficacy trial. Lancet Oncol 6(5):271-278

20. Koshiol J, Schroeder JC, Jamieson DJ et al (2006) Time to clearance of human papillomavirus (HPV) infection by HPV type in HIV-seropositive and HIV-seronegative women. Intl J Cancer 119(7):1623-1629

21. Heard I, Schmitz V, Costagliola D, Orth G, Kazatchkine MD (1998) Early regression of cervical lesions in HIV-seropositive women receiving highly active antiretroviral therapy. AIDS 12:1459-1464

22. Minkoff H, Ahdieh L, Massad LS et al (2001) The effect of highly active antiretroviral therapy on cervical cytologic changes associated with oncogenic HPV among HIV-infected women. AIDS 15:2157-2164
23. Lillo FB, Ferrari D, Veglia F et al (2001) Human papillomavirus infection and associated cervical disease in human immunodeficiency virus-infected women: effect of highly active antiretroviral therapy. J Infect Dis 184:547-551

24. De Vuyst H, Lillo F, Broutet N, Smith JS (2008) HIV, HPV and cervical cancer and neoplasia in the era of HAART, and the opportunities for intervention during the post-partum. Eur $\mathrm{J}$ Cancer 17(6):545-554

25. Firnhaber CS, Zungu K, Williamson AL et al (2009) A high prevalence of high risk human papillomavirus and cervical dysplasia in HIV seropositive women in an urban South African Cervical Cancer Cohort (SACCC). Cytologica Acta 53:10-18

26. Solomon D, Davey D, Kurman R et al (2002) The 2001 Bethesda system terminology for reporting results of cervical cytology. JAMA 287:2114-2119

27. Tshabalala-Msimang ME, Mbewu A, Simelela N, et al. (2003) Operational plan for comprehensive HIV and AIDS care, management and treatment for South Africa. 19 November 2003

28. Muñoz N, Bosch FX, de Sanjosé S et al (2003) Epidemiologic classification of human papillomavirus types associated with cervical cancer. N Engl J Med 348:518-527

29. Barros AJ, Hirakata VN (2003) Alternatives for logistic regression in cross-sectional studies: an empirical comparison of models that directly estimate the prevalence ratio. BMC Med Res Methodol 3:21

30. Denny L, Boa R, Williamson AL, Allan B, Hardie D, Stan R, Myer I (2008) Human papillomavirus infection and cervical disease in human immunodeficiency virus-1-infected women. Obstet Gynecol 111(6):1380-1387

31. Parham GP, Sahasrabuddhe VV, Mwanahamuntu MH, Shepherd BE, Hicks ML, Stringer EM (2006) Prevalence and predictors of squamous intraepithelial lesions of the cervix in HIV-infected women in Lusaka, Zambia. Gynecol Oncol 103(3):1017-1022

32. Six C, Heard I, Bergeron C et al (1998) Comparative prevalence, incidence and short-term prognosis of cervical squamous intraepithelial lesions amongst HIV-positive and HIV-negative women. AIDS 12:1047-1056

33. Delmas MC, Larsen C, van Benthem B et al (2000) Cervical squamous intraepithelial lesions in HIV-infected women: prevalence, incidence and regression. European study group on natural history of HIV infection in women. AIDS 14:1775-1784

34. Chirenje ZM, Loeb L, Mwale M, Nyamapfeni P, Kamba M, Padian N (2002) Association of cervical SIL and HIV 1 infection on Zimbabwean women in an HIV/STI prevention study. Int $\mathbf{J}$ STD AIDS 13:765-768

35. Cronje HS (2004) Screening for cervical cancer in developing countries. Int J Gynaecol Obstet 84:101-108

36. Sankaranarayanan R, Budukh AM, Rajkumar R (2001) Effective screening programmes for cervical cancer in low- and middle-income developing countries. Bull World Health Organ 79(10):954-962

37. Strickler HD, Burk RD, Fazzari M, Levine AM et al (2005) Natural history and possible reactivation of human papillomavirus in human immunodeficiency virus-positive women. J Natl Cancer Inst 97:577-586

38. Schuman P, Ohmit SE, Klein RS et al (2003) Longitudinal study of cervical squamous intraepithelial lesions in human immunodeficiency virus (HIV)-seropositive and at-risk HIVseronegative women. J Infect Dis 188:128-136

39. Jamieson DJ, Duerr A, Burk R et al (2002) HIV epidemiology research study (HERS) group. Characterization of genital human papillomavirus infection in women who have or who are at risk of having HIV infection. Am J Obstet Gynecol 186:21-27

40. Conley LJ, Ellerbrock TV, Bush TJ, Chiasson MA, Sawo D, Wright TC (2002) HIV-1 infection and risk of vulvovaginal and perianal condylomata acuminata and intraepithelial neoplasia: a prospective cohort study. Lancet 359:108-113 
41. Winer RL, Hughes JP, Feng Q et al (2006) Condom use and the risk of genital human papillomavirus infection in young women. N Engl J Med 354:2642-2654
42. Holmes KK, Levine R, Weaver M (2004) Effectiveness of condoms in preventing sexually transmitted infections. Bull World Health Organ 82(6):454-461 